\title{
Controllable switching of vortex chirality in magnetic nanodisks by a field pulse
}

\author{
Yuri Gaididei, ${ }^{1}$ Denis D. Sheka, ${ }^{2}$, and Franz G. Mertens ${ }^{3}$ \\ ${ }^{1}$ Institute for Theoretical Physics, 03143 Kiev, Ukraine \\ ${ }^{2}$ National Taras Shevchenko University of Kiev, 03127 Kiev, Ukraine \\ ${ }^{3}$ Physics Institute, University of Bayreuth, 95440 Bayreuth, Germany
}

(Dated: October 23, 2007)

\begin{abstract}
We propose a way of fast switching the chirality in a magnetic nanodisk by applying a field pulse. To break the symmetry with respect to clockwise or counterclockwise chirality a mask is added by which an inhomogeneous field influences the vortex state of a nanodisk. Using numerical spinlattice simulations we demonstrate that chirality can be controllably switched by a field pulse, whose intensity is above some critical value. A mathematical definition for the chirality of an arbitrary shaped particle is proposed.
\end{abstract}

PACS numbers: 75.10.Hk, 75.70.Ak, 75.40.Mg, 05.45.-a

In the last few years the nonlinear dynamics of magnetic nanostructures has been extensively studied in order to develop useful devices for storage and transmission of information. Magnetic nanodots with their vortex ground state show a considerable promise as candidates for high density magnetic storage and high speed magnetic random access memory [1]. The vortex state disks are characterized by the following conserved quantities, which can be associated with a bit of information: the polarity, the sense of the vortex core magnetization direction (up or down) and the chirality or handedness, the sense of magnetization rotation (clockwise or counterclockwise). Until recently the main efforts went into the control of vortex polarity and vortex motion inside the nanodots. The polarity switching under the action of an ac magnetic field was predicted theoretically [2] and was recently observed experimentally in Permalloy nanodisks by using short bursts of an ac magnetic field [3. The polarity switching under the action of a dc electrical current was predicted theoretically [4] and observed experimentally quite recently [5]. The main features of the polarity switching mechanism were clarified in Refs. 3, 6, 7, 8, 9, 10, where it was shown that no matter whether the switching is induced by a magnetic field or by an electrical current it involves creation of a vortex-antivortex pair and subsequent annihilation of the new-born anti-vortex with the original vortex.

In standard experiments with in-plane field and circular nanodisks the vortex chirality is not under control because of the high symmetry of the system [11. However, the chirality is known to be controlled by introducing an asymmetry in the particle shape, like flat edges of the disk [12, 13, rings with asymmetrical inner holes [14], and elliptical dots [15, 16. Quite recently it was demonstrated [17] that the vortex chirality can be switched by injecting a current pulse with appropriate amplitude, polarity and duration. This was achieved in a multilayer structure with two different magnetic layers, each being in a vortex state.

The aim of the present Letter is to show that the vor-

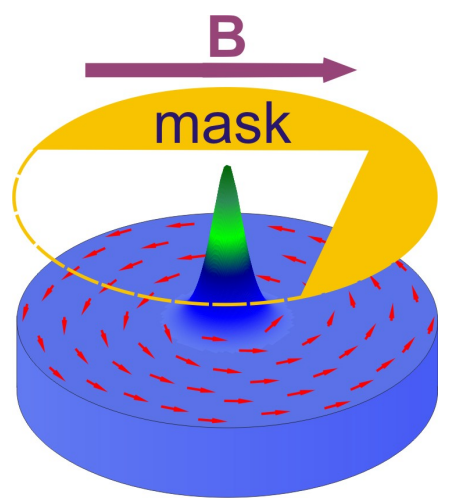

FIG. 1: (Color online) Schematic of the structure. The mask is added to create an inhomogeneous magnetic field, which breaks the symmetry and separates one chirality direction.

tex chirality can be controllably switched by applying a magnetic field pulse in a circular magnetic disk. We use the disk-shaped particle in a vortex state, see Fig. 1. A magnetic field is applied in the disk plane, which moves the vortex away from the disk center. If the homogeneous DC field exceeds the value $b_{\text {an }}$, the vortex is annihilated, and finally the vortex state switches to the monodomain state 18. After that the field is switched off, and a vortex enters the system, however the chirality of the new-born vortex could be either clockwise or counterclockwise [11. To control the chirality of nucleated vortex one needs to break the symmetry with respect to chirality. We propose to break it by inhomogeneous field, which can be simply realized, e.g. by using a mask, which shields a part of the disk from the field influence, see Fig. 1 .

To realize this program let us consider the dynamics of classical lattice spins, described by the Landau-LifshitzGilbert equations

$$
\frac{\mathrm{d} \boldsymbol{S}_{\boldsymbol{n}}}{\mathrm{d} \tau}=-\boldsymbol{S}_{\boldsymbol{n}} \times \frac{\partial \mathcal{H}}{\partial \boldsymbol{S}_{\boldsymbol{n}}}-\alpha \boldsymbol{S}_{\boldsymbol{n}} \times \frac{\mathrm{d} \boldsymbol{S}_{\boldsymbol{n}}}{\mathrm{d} \tau},
$$

where $\boldsymbol{S}_{\boldsymbol{n}}$ is a unit vector which determines the spin direction at the lattice point $\boldsymbol{n}$, the lattice constant is cho- 

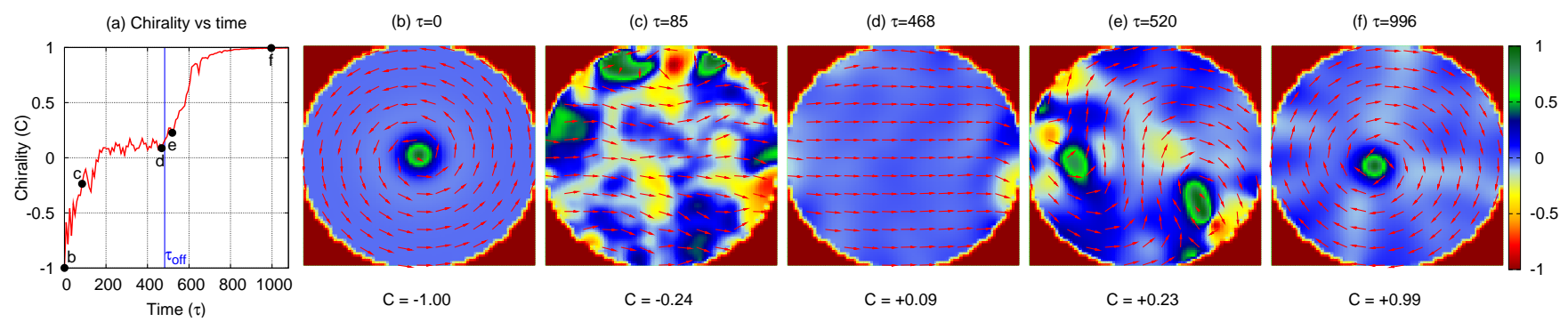

FIG. 2: (Color online) Time evolution of the vortex chirality switching process from simulations with a magnetic field pulse of amplitude $b=0.2$ and duration $\tau=480$. (a) The vortex chirality as a function of time. (b)-(f) Snapshot images illustrating in-plane magnetization distributions at different stages of the vortex dynamics.

sen as the unity length. The dimensionless time $\tau=\omega_{0} t$ with $\omega_{0}=4 \pi \gamma M_{S}$ and $M_{S}$ being the saturation magnetization; $\alpha \ll 1$ is a damping coefficient. The Hamiltonian $\mathcal{H}$ :

$$
\begin{aligned}
\mathcal{H} & =-\frac{\ell^{2}}{2} \sum_{(\boldsymbol{n}, \boldsymbol{\delta})} \boldsymbol{S}_{\boldsymbol{n}} \cdot \boldsymbol{S}_{\boldsymbol{n}+\boldsymbol{\delta}}-\sum_{\boldsymbol{n}} \boldsymbol{b} \cdot \boldsymbol{S}_{\boldsymbol{n}} \\
& +\frac{1}{8 \pi} \sum_{\substack{\boldsymbol{n}, \boldsymbol{n}^{\prime} \\
\boldsymbol{n} \neq \boldsymbol{n}^{\prime}}} \frac{\boldsymbol{S}_{\boldsymbol{n}} \cdot \boldsymbol{S}_{\boldsymbol{n}^{\prime}}-3\left(\boldsymbol{S}_{\boldsymbol{n}} \cdot \boldsymbol{e}_{\boldsymbol{n} \boldsymbol{n}^{\prime}}\right)\left(\boldsymbol{S}_{\boldsymbol{n}^{\prime}} \cdot \boldsymbol{e}_{\boldsymbol{n} \boldsymbol{n}^{\prime}}\right)}{\left|\boldsymbol{n}-\boldsymbol{n}^{\prime}\right|^{3}}
\end{aligned}
$$

consists of exchange, Zeeman and dipolar terms. Here $\ell=\sqrt{A /\left(4 \pi M_{S}^{2}\right)}$ is the exchange length ( $A$ is the exchange constant), the vector $\delta$ connects nearest neighbors, and $\boldsymbol{e}_{\boldsymbol{n} \boldsymbol{n}^{\prime}} \equiv\left(\boldsymbol{n}-\boldsymbol{n}^{\prime}\right) /\left|\boldsymbol{n}-\boldsymbol{n}^{\prime}\right|$ is a unit vector. The dimensionless magnetic dc field $\boldsymbol{b}=\boldsymbol{B} / 4 \pi M_{S}$.

In the continuum description, the spin dynamics is described by a magnetization unit vector $\boldsymbol{m}=-\left\langle\boldsymbol{S}_{\boldsymbol{n}}\right\rangle$. Usually the chirality is introduced as the sense of a magnetization rotation: either clockwise or counterclockwise around some direction $\boldsymbol{N}$. To describe the chirality as a continuous quantity we introduce the following definition:

$$
C=\frac{1}{L} \int_{\sigma}[\nabla \times \boldsymbol{m}] \cdot \mathrm{d} \boldsymbol{\sigma}=\frac{1}{L} \int_{\partial \sigma} \boldsymbol{m} \cdot \mathrm{d} \boldsymbol{\tau}
$$

where $\boldsymbol{\sigma}=\sigma \boldsymbol{e}_{N}$ is a surface, perpendicular to $\boldsymbol{e}_{N}$, and $L$ is the length of a contour, which bounds a surface $\sigma$. For a disk-shaped particle of radius $R$ with in-plane magnetization distribution, described by the angular variable $\phi=$ $\arctan \left(m^{y} / m^{x}\right)$, the chirality $C=\frac{1}{2 \pi} \int_{0}^{2 \pi} \sin (\phi-\chi) \mathrm{d} \chi$ around the circle of radius $R$, where $\chi$ is a polar angle in the disk plane. The in-plane magnetization distribution in the vortex state nanodisk is described by the formula $\phi=\chi+C \pi / 2$.

The physical idea of the chirality switching is very simple. In an external dc magnetic field $b$, which is stronger that some annihilation field $b_{\text {an }}$, but below the saturation field $b_{\text {sat }}$, the monodomain state appears. However, if the field $b$ is inhomogeneous, the monodomain state is nonuniform. By choosing the shape of the field, one can create this intermediate monodomain state with a certain nonzero chirality. After that, if we switch off the field, the vortex will be nucleated with a predefined chirality.

To study the vortex dynamics, we have performed numerical simulations of the discrete spin-lattice Eqs. (1). We consider the case of thin nanodots where the magnetization does not depend on the z-coordinate, the details of the method are described in Ref. [19. We have integrated numerically the set of Eqs. (1) over a square lattice using the fourth-order Runge-Kutta scheme with time step $\Delta \tau=0.01$. The lattice is bounded by a circle of diameter $2 R$. In most of the simulations the system diameter $2 R=50$, the thickness $h=10$, other parameters: $\ell=1.33, \alpha=0.01$. We applied an inhomogeneous field along the $x$ axis by the shape $b(x, y)=b \Theta(\xi-x) \Theta(\xi-y)$ with $\Theta(x)$ being the Heaviside step function. We choose $\xi /(2 R)=0.85$. Such a field can be realized by a mask which covers a part of the disk, see Fig. 1 .

The temporal evolution of the chirality is presented in Fig. 2(a). Initially the chirality was $C=-1$ (counterclockwise), see Fig. 2(b). Under the action of the field pulse the vortex is pushed away in the direction of the north pole of the disk, which is accompanied by a decrease of the absolute value of the chirality, see Fig. 2(c). The next monodomain stage is presented in Fig. 2(d). The vortex is already annihilated, but the total chirality takes a nonzero (positive) value due to the mask influence. After that the field pulse is switched off, and the new vortex enters the disk from the south pole with a predefined (positive) chirality, see Fig. 2(e). This vortex quickly moves to the disk center, and finally the chirality is $C=+1$ (clockwise), see Fig. 2(f). The switching process takes place in a wide range of parameters of the field pulse, when the field intensity $b>b_{\text {an }}$, see Fig. 3 .

Using typical parameters for permalloy disks [20], one can estimate the exchange length $\ell=5.3 \mathrm{~nm}$, which corresponds to a particle with $2 R=200 \mathrm{~nm}$ and $h=40$ $\mathrm{nm}$. The typical time unit $1 / \omega_{0}=50 \mathrm{ps}$ and the field unit $4 \pi M_{S}=10 \mathrm{kOe}$. The typical switching field intensity is about 140 Oe and the typical switching time is about $7 \mathrm{~ns}$.

To summarize, we have studied the chirality switching 
(a) $b=0.160$

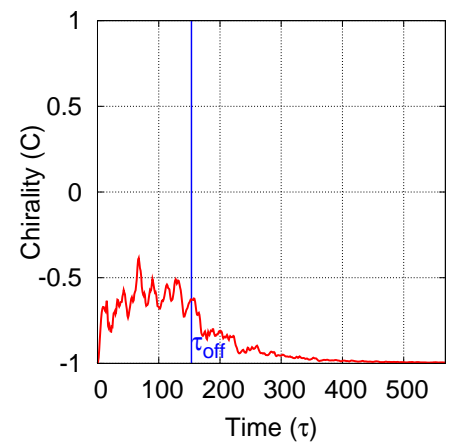

(c) $b=0.200$

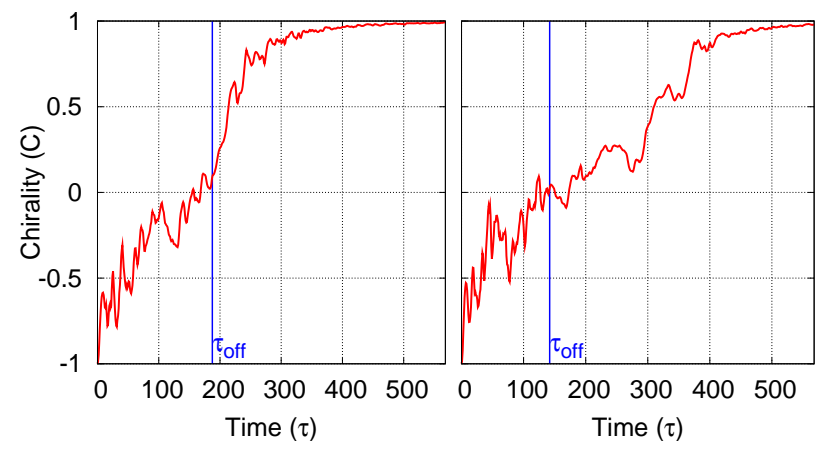

FIG. 3: (Color online) Time dependence of the vortex chirality for different amplitudes of field pulses: (a) without switching, (b)-(d) with chirality switching. The switching occurs, when the field amplitude exceeds the critical value $b_{\text {an }} \approx 0.163$.

under the action of a dc magnetic field. In contrast to previous studies on the chirality control, we showed that the switching can be managed in a pure disk nanoparticle: to break the symmetry the usage of the mask is proposed. Usually the chirality is considered as either clockwise or counterclockwise. By considering the chirality as a continuous quantity, we were able to control the switching process: the key point is an intermediate state with a nonzero chirality. We consider here only one possible realization of the chirality switching with a very simple mask. We expect that the usage of a mask will be prominent for different applications of nonlinear magnetization dynamics in nanoparticles.

The authors thank H. Stoll and M. Fähnle for stimulating discussions. D.S., Yu.G. thank the University of Bayreuth, where this work was performed, for kind hospitality, and acknowledge the support from DLR grant No. UKR 05/055. D.S. acknowledges the support from the Alexander von Humboldt-Foundation and the grant No. F25.2/098 from the Fundamental Researches State Fund of Ukraine.
* Corresponding author. Electronic address: denis_sheka@univ.kiev.ua

[1] R. P. Cowburn, J. Magn. Magn. Mater. 242245, 505 (2002), URL http://dx.doi.org/10.1016/ S0304-8853(01)01086-1.

[2] Y. Gaididei, T. Kamppeter, F. G. Mertens, and A. R. Bishop, Phys. Rev. B 61, 9449 (2000), URL http:// link.aps.org/abstract/PRB/v61/p9449

[3] V. B. Waeyenberge, A. Puzic, H. Stoll, K. W. Chou, T. Tyliszczak, R. Hertel, M. Fahnle, H. Bruckl, K. Rott, G. Reiss, et al., Nature 444, 461 (2006), ISSN 0028-0836, URL http://dx.doi .org/10.1038/nature05240

[4] J.-G. Caputo, Y. Gaididei, F. G. Mertens, and D. D. Sheka, Phys. Rev. Lett. 98, 056604 (pages 4) (2007), URL http://link.aps .org/abstract/PRL/v98/ e056604

[5] K. Yamada, S. Kasai, Y. Nakatani, K. Kobayashi, H. Kohno, A. Thiaville, and T. Ono, Nat Mater 6, 270 (2007), ISSN 1476-1122, URL http://dx.doi.org/10. $1038 /$ nmat 1867 .

[6] Q. F. Xiao, J. Rudge, B. C. Choi, Y. K. Hong, and G. Donohoe, Applied Physics Letters 89, 262507 (pages 3) (2006), URL http://link.aip.org/link/ ?APL/89/262507/1.

[7] R. Hertel, S. Gliga, M. Fahnle, and C. M. Schneider, Phys. Rev. Lett. 98, 117201 (pages 4) (2007), URL http: //link.aps.org/abstract/PRL/v98/e117201

[8] K.-S. Lee, K. Y. Guslienko, J.-Y. Lee, and S.-K. Kim, Ultrafast vortex-core reversal dynamics in ferromagnetic nanodots (2007), cond-mat/0703538, URL http://www . arxiv.org/abs/cond-mat/0703538

[9] V. P. Kravchuk, D. D. Sheka, Y. Gaididei, and F. G. Mertens, Controlled vortex core switching in a magnetic nanodisk by a rotating field (2007), arXiv:0705.2046, URL http: //arxiv .org/abs/0705.2046.

[10] D. D. Sheka, Phys. Rev. B 75, 107401 (pages 2) (2007), URL http://link .aps.org/abstract/PRB/v75/ e107401.

[11] M. Schneider, H. Hoffmann, and J. Zweck, Appl. Phys. Lett. 77, 2909 (2000), URL http://link.aip.org/ link/?APL/77/2909/1.

[12] M. Schneider, H. Hoffmann, and J. Zweck, Appl. Phys. Lett. 79, 3113 (2001), URL http://link.aip.org/ link/?APL/79/3113/1.

[13] T. Kimura, Y. Otani, H. Masaki, T. Ishida, R. Antos, and J. Shibata, Appl. Phys. Lett. 90, 132501 (pages 3) (2007), URL http://link. aip.org/link/?APL/90/132501/1.

[14] A. Subramani, D. Geerpuram, V. Baskaran, J. Friedlund, and V. Metlushko, Physica C: Superconductivity 437-438, 293 (2006), URL http://www.sciencedirect. com/science/article/B6TVJ-4J624M1-5/2/ 6a578dde6b9baa5c53e8a7655800cd16

[15] P. Vavassori, N. Zaluzec, V. Metlushko, V. Novosad, B. Ilic, and M. Grimsditch, Phys. Rev. B 69, 214404 (pages 6) (2004), URL http://link.aps.org/abstract/ $\mathrm{PRB} / \mathrm{v} 69 / \mathrm{e} 214404$

[16] V. Mironov, B. Gribkov, A. Fraerman, S. Gusev, S. Vdovichev, I. Karetnikova, I. Nefedov, and I. Shereshevsky, J. Magn. Magn. Mater. 312, 153 (2007), URL http://www.sciencedirect. com/science/article/B6TJJ-4M6S933-1/2/ 
339dcee0d40dbfe9b27888e9b44a70f 9 .

[17] B. C. Choi, J. Rudge, E. Girgis, J. Kolthammer, Y. K. Hong, and A. Lyle, Appl. Phys. Lett. 91, 022501 (pages 3) (2007), URL http://link.aip.org/link/ ?APL/91/022501/1

[18] A. Hubert and R. Schäfer, Magnetic domains (SpringerVerlag, Berlin, 1998).

[19] J.-G. Caputo, Y. Gaididei, V. P. Kravchuk, F. G.
Mertens, and D. D. Sheka, Effective anisotropy of thin nanomagnets: beyond the surface anisotropy approach (2007), arXiv:0705.1555, URL http://arxiv.org/abs/ 0705.1555

[20] For the Py particle the exchange constant $A=$ $2.6 \mu \mathrm{erg} / \mathrm{cm}$, the saturation magnetization $M_{S}=0.86$ $\mathrm{kG}$. 